PROCEEDINGS OF THE

AMERICAN MATHEMATICAL SOCIETY

Volume 132, Number 3, Pages 885-890

S 0002-9939(03)07175-2

Article electronically published on July 7, 2003

\title{
WIGNER-YANASE SKEW INFORMATION VS. QUANTUM FISHER INFORMATION
}

\author{
SHUNLONG LUO
}

(Communicated by David R. Larson)

\begin{abstract}
Among concepts describing the information contents of quantum mechanical density operators, both the Wigner-Yanase skew information and the quantum Fisher information defined via symmetric logarithmic derivatives are natural generalizations of the classical Fisher information. We will establish a relationship between these two fundamental quantities and show that they are comparable.
\end{abstract}

\section{INTRODUCTION}

In the study of information contents of distributions (quantum mechanical density operators), Wigner and Yanase [10] introduced the quantity $I_{W}(\rho, H)=$ $\frac{1}{2} \operatorname{tr}\left(D_{H} \rho^{1 / 2}\right)^{2}$, which they called skew information, to measure the information content of the density operator $\rho$ with respect to the fixed self-adjoint operator $H$ (which may be interpreted as a Hamiltonian, a momentum, or other conserved quantity). Here $D_{H}$ is the inner differentiation with respect to $H$ defined as $D_{H} x=i[x, H]=i(x H-H x)$. Alternatively, $I_{W}(\rho, H)$ may be interpreted as a measure of non-commutativity between $\rho$ and $H$ [3]. Wigner and Yanase argued and proved that this quantity satisfied all the desirable intuitive requirements of an information measure [10, [11]. This notion of skew information was further generalized by Connes and Stormer [3] in a general von Neumann algebra setting. It played a crucial role when they proved the homogeneity of state spaces of $\mathrm{III}_{1}$ von Neumann algebras.

In fact, the notion of skew information is very similar to the well-known notion of Fisher information originated from statistical inference [5], [6]. Recall that the Fisher information of a parameterized family of probability densities $\left\{p_{\theta}: \theta \in \mathbb{R}\right\}$ on $\mathbb{R}$ is defined as

$$
\begin{aligned}
I_{F}\left(p_{\theta}\right) & =\int_{\mathbb{R}}\left(\frac{\partial}{\partial \theta} p_{\theta}^{1 / 2}(x)\right)^{2} d x \\
& =\frac{1}{4} \int_{\mathbb{R}}\left(\frac{\partial}{\partial \theta} \log p_{\theta}(x)\right)^{2} p_{\theta}(x) d x .
\end{aligned}
$$

Received by the editors January 23, 2002 and, in revised form, November 16, 2002.

2000 Mathematics Subject Classification. Primary 62B10, 94A17; Secondary 46L30, 46L60.

Key words and phrases. Fisher information, density operators, von Neumann-Landau equation, skew information, quantum Fisher information.

This work was supported by the NSF of China, Grant No. 10131040. 
In particular, when $p_{\theta}(x)=p(x-\theta)$, by the translation invariance of the Lebesgue integral, we have

$$
\begin{aligned}
I_{F}\left(p_{\theta}\right) & =\int_{\mathbb{R}}\left(\frac{\partial}{\partial x} p^{1 / 2}(x)\right)^{2} d x \\
& =\frac{1}{4} \int_{\mathbb{R}}\left(\frac{\partial}{\partial x} \log p(x)\right)^{2} p(x) d x .
\end{aligned}
$$

Thus, in this circumstance, $I_{F}\left(p_{\theta}\right)$ is independent of $\theta$, and we can denote $I_{F}\left(p_{\theta}\right)$ simply by $I_{F}(p)$. It is the Fisher information of $p$ with respect to the location parameter. The notion of Fisher information plays a crucial role in the geometrical approach to statistics [1, and it is enjoying increasing popularity in the informational approach to physics 7 .

While it is remarkable that Chentsov [2] characterized the metric induced by the Fisher information as the unique (up to a constant factor) monotone Riemann metric (that is, metric contracts under any stochastic mapping) on the class of probability densities (classical or commutative case), it is equally remarkable that Petz [9] determined all monotone Riemann metrics on density matrices (quantum or noncommutative case). In particular, in an operator setting (quantum case), the notion of Fisher information has many natural generalizations due to non-commutativity, in sharp contrast to the uniqueness in the commutative case. Among the various generalizations, there are two distinguished ones. The first arises when one formally generalizes the expression $I_{F}\left(p_{\theta}\right)=\int_{\mathbb{R}}\left(\frac{\partial}{\partial \theta} p_{\theta}^{1 / 2}(x)\right)^{2}$ defined by Eq. (1). Replacing the integration by trace, the parameterized probabilities $p_{\theta}$ by a parameterized density operator $\rho_{\theta}$ on some Hilbert space, and the ordinary differential $\frac{\partial}{\partial \theta}$ by the quantum (inner) differential $D_{H}$ in non-commutative geometry ( $H$ is a fixed self-adjoint operator) [4], we can heuristically define

$$
I_{W}\left(\rho_{\theta}\right)=\operatorname{tr}\left(D_{H} \rho_{\theta}^{1 / 2}\right)^{2} .
$$

In particular, when $\rho_{\theta}=e^{-i \theta H} \rho e^{i \theta H}, I_{W}\left(\rho_{\theta}\right)$ is independent of the parameter $\theta$ as will be shown in Section 2. In this circumstance, $I_{W}\left(\rho_{\theta}\right)$ is precisely the WignerYanase skew information $I_{W}(\rho, H)=\frac{1}{2} \operatorname{tr}\left(D_{H} \rho^{1 / 2}\right)^{2}$, which corresponds to the generalization of Eq. (3).

The second natural generalization of the classical Fisher information arises when one formally generalizes the expression $I_{F}\left(p_{\theta}\right)=\frac{1}{4} \int_{\mathbb{R}}\left(\frac{\partial}{\partial \theta} \log p_{\theta}(x)\right)^{2} p_{\theta}(x) d x$ defined by Eq. (2). This was actually first done in a quantum estimation setting [8]. To see how this happens, note that in a symmetric form,

$$
\frac{\partial}{\partial \theta} p_{\theta}=\frac{1}{2}\left(\frac{\partial}{\partial \theta} \log p_{\theta} \cdot p_{\theta}+p_{\theta} \cdot \frac{\partial}{\partial \theta} \log p_{\theta}\right) .
$$

In Eq. (2), replacing the integration by trace, $p_{\theta}$ by $\rho_{\theta}$, and the logarithmic derivative $\frac{\partial}{\partial \theta} \log p_{\theta}$ by the symmetric logarithmic derivative $L_{\theta}$ determined by

$$
\frac{\partial}{\partial \theta} \rho_{\theta}=\frac{1}{2}\left(L_{\theta} \rho_{\theta}+\rho_{\theta} L_{\theta}\right), \quad \theta \in \mathbb{R},
$$

we come to the quantum Fisher information (derived via the symmetric logarithmic derivative)

$$
I_{F}\left(\rho_{\theta}\right)=\frac{1}{4} \operatorname{tr}\left(L_{\theta}^{2} \rho_{\theta}\right)
$$


This generalization plays an important role in quantum estimation [8]. In particular, when $\rho_{\theta}=e^{-i \theta H} \rho e^{i \theta H}, I_{F}\left(\rho_{\theta}\right)$ is independent of the parameter $\theta$ as will be shown in Section 2, and in this circumstance, $I_{F}\left(\rho_{\theta}\right)$ coincides with $I_{F}(\rho, H)=$ $\frac{1}{4} \operatorname{tr}\left(\rho L^{2}\right)$, which corresponds to the generalization of Eq. (4). Here $L$ is a quantum analogue of the symmetric logarithmic derivative determined by

$$
D_{H} \rho=\frac{1}{2}(L \rho+\rho L) \text {. }
$$

Of course, one can also write $\frac{\partial}{\partial \theta} p_{\theta}=p_{\theta} \cdot \frac{\partial}{\partial \theta} \log p_{\theta}$, and consider a version of quantum Fisher information defined via the right logarithmic derivative. We do not pursue this case here.

Now a natural question arises: How are these two generalizations of the classical Fisher information, $I_{W}(\rho, H)$ and $I_{F}(\rho, H)$, related? We shall show that

$$
I_{W}(\rho, H) \leq I_{F}(\rho, H) \leq 2 I_{W}(\rho, H) .
$$

In fact, using resolution of identity and a representation diagonalizing the density operator $\rho$, we are able to compute explicitly both $I_{W}(\rho, H)$ and $I_{F}(\rho, H)$, from which the comparing result follows. The proof is facilitated by the formal manipulation of the associativity of Dirac'c bras, kets and operators.

\section{Skew information vs. QUANTUm Fisher information}

Let $H$ be a fixed observable (Hermitian operator) on some Hilbert space $\mathcal{H}$ of quantum states representing a certain conserved quantity. Let $\rho$ be a density operator on $\mathcal{H}$. Let $\rho_{\theta}=e^{-i \theta H} \rho e^{i \theta H}$ be the evolution of $\rho$ generated by $H$. Here $\theta \in \mathbb{R}$ is a (temporal or spatial) parameter, and $H$ may be interpreted as the generator of the temporal shift or the spatial displacement. Clearly, $\rho_{\theta}$ satisfies the von Neumann-Landau equation

$$
i \frac{\partial \rho_{\theta}}{\partial \theta}=H \rho_{\theta}-\rho_{\theta} H, \quad \theta \in \mathbb{R} .
$$

Theorem 1. Under the above assumptions, we have

(1) $I_{W}\left(\rho_{\theta}\right)=I_{W}(\rho, H), \forall \theta \in \mathbb{R}$. That is, $I_{W}\left(\rho_{\theta}\right)$ is independent of the parameter $\theta$, and coincides with the Wigner-Yanase skew information $I_{W}(\rho, H)$.

(2) $I_{F}\left(\rho_{\theta}\right)=I_{F}(\rho, H), \forall \theta \in \mathbb{R}$. That is, $I_{F}\left(\rho_{\theta}\right)$ is independent of the parameter $\theta$, and coincides with $I_{F}(\rho, H)$.

Proof. (1) The conclusion follows from the cycle property of trace and

$$
D_{H} \rho_{\theta}^{1 / 2}=e^{-i \theta H}\left(D_{H} \rho^{1 / 2}\right) e^{i \theta H} .
$$

(2) By Eqs. (6) and (7), we have

$$
i\left(\rho_{\theta} H-H \rho_{\theta}\right)=\frac{1}{2}\left(L_{\theta} \rho_{\theta}+\rho_{\theta} L_{\theta}\right)
$$

which implies

$$
i(\rho H-H \rho)=\frac{1}{2}(L \rho+\rho L) \text {. }
$$

Here $L=e^{i \theta H} L_{\theta} e^{-i \theta H}$. Clearly, $L$ is independent of the parameter $\theta$ since it is the unique solution of Eq. (8) when $H$ and $\rho$ (which are independent of $\theta$ ) are fixed. By the cyclic property of trace, we have

$$
I_{F}\left(\rho_{\theta}\right)=\frac{1}{4} \operatorname{tr}\left(\rho_{\theta} L_{\theta}^{2}\right)=\frac{1}{4} \operatorname{tr}\left(e^{-i \theta H} \rho e^{i \theta H} L_{\theta}^{2}\right)=\frac{1}{4} \operatorname{tr}\left(\rho e^{i \theta H} L_{\theta}^{2} e^{-i \theta H}\right)=\frac{1}{4} \operatorname{tr}\left(\rho L^{2}\right) .
$$

Theorem 2. $I_{W}(\rho, H) \leq I_{F}(\rho, H) \leq 2 I_{W}(\rho, H)$. 
Proof. We need only to treat the case when $\rho$ is non-degenerate. When $\rho$ is degenerate, simple modification of the following argument yields the same conclusion. Thus suppose $\rho$ has the orthogonal spectral representation

$$
\rho=\sum_{m} \lambda_{m}\left|\psi_{m}\right\rangle\left\langle\psi_{m}\right|
$$

Here $\left\{\lambda_{m}\right\}$ are the different (positive) eigenvalues of $\rho$, and $\left\{\left|\psi_{m}\right\rangle\right\}$ constitute an orthonormal base. We have used Dirac's notation, $\left|\psi_{m}\right\rangle\left\langle\psi_{m}\right|$ stands for the projection on the ket vector $\left|\psi_{m}\right\rangle$. For any operator $A,\left\langle\psi_{m}|A| \psi_{n}\right\rangle$ stands for $\left\langle\psi_{m}, A \psi_{n}\right\rangle$. The inner product is linear in the second variable. The advantage of using Dirac's notation will be clear in the subsequent proof. By virtue of the orthonormal base $\left\{\left|\psi_{m}\right\rangle\right\}$, we have the resolution of identity

$$
\mathbf{1}=\sum_{m}\left|\psi_{m}\right\rangle\left\langle\psi_{m}\right|
$$

Here 1 stands for the identity operator on $\mathcal{H}$.

Now we compute the skew information $I_{W}(\rho, H)$. Since

$$
\begin{aligned}
\operatorname{tr}\left(\rho H^{2}\right) & =\sum_{m}\left\langle\psi_{m}\left|\rho H^{2}\right| \psi_{m}\right\rangle \\
& =\sum_{m} \lambda_{m}\left\langle\psi_{m}\left|H^{2}\right| \psi_{m}\right\rangle \quad \text { (by Eq. (9)) } \\
& =\sum_{m} \lambda_{m}\left\langle\psi_{m}\left|H \sum_{n}\right| \psi_{n}\right\rangle\left\langle\psi_{n}|H| \psi_{m}\right\rangle \quad \text { (by Eq. (10)) } \\
& =\sum_{m, n} \lambda_{m}\left\langle\psi_{m}|H| \psi_{n}\right\rangle\left\langle\psi_{n}|H| \psi_{m}\right\rangle \\
& =\sum_{m, n} \lambda_{m}\left|\left\langle\psi_{m}|H| \psi_{n}\right\rangle\right|^{2} .
\end{aligned}
$$

Symmetrically, we also have

$$
\operatorname{tr}\left(\rho H^{2}\right)=\sum_{m, n} \lambda_{n}\left|\left\langle\psi_{m}|H| \psi_{n}\right\rangle\right|^{2} .
$$

Consequently,

$$
\operatorname{tr}\left(\rho H^{2}\right)=\sum_{m, n} \frac{\lambda_{m}+\lambda_{n}}{2}\left|\left\langle\psi_{m}|H| \psi_{n}\right\rangle\right|^{2} .
$$

On the other hand,

$$
\begin{aligned}
\operatorname{tr}\left(H \rho^{1 / 2} H \rho^{1 / 2}\right) & =\sum_{m}\left\langle\psi_{m}\left|H \rho^{1 / 2} H \rho^{1 / 2}\right| \psi_{m}\right\rangle \\
& =\sum_{m} \lambda_{m}^{1 / 2}\left\langle\psi_{m}\left|H \rho^{1 / 2} H\right| \psi_{m}\right\rangle \\
& =\sum_{m, n} \lambda_{m}^{1 / 2}\left\langle\psi_{m}\left|H \rho^{1 / 2}\right| \psi_{n}\right\rangle\left\langle\psi_{n}|H| \psi_{m}\right\rangle \\
& =\sum_{m, n} \lambda_{m}^{1 / 2} \lambda_{n}^{1 / 2}\left\langle\psi_{m}|H| \psi_{n}\right\rangle\left\langle\psi_{n}|H| \psi_{m}\right\rangle \\
& =\sum_{m, n} \lambda_{m}^{1 / 2} \lambda_{n}^{1 / 2}\left|\left\langle\psi_{m}|H| \psi_{n}\right\rangle\right|^{2} .
\end{aligned}
$$


Combining Eqs. (12) and (13), we have

$$
\begin{aligned}
I_{W}(\rho, H) & =\operatorname{tr}\left(\rho H^{2}\right)-\operatorname{tr}\left(H \rho^{1 / 2} H \rho^{1 / 2}\right) \\
& =\frac{1}{2} \sum_{m, n}\left(\lambda_{m}^{1 / 2}-\lambda_{n}^{1 / 2}\right)^{2}\left|\left\langle\psi_{m}|H| \psi_{n}\right\rangle\right|^{2} .
\end{aligned}
$$

Next, we evaluate the quantum Fisher information $I_{F}(\rho, H)$. Since

$$
i(\rho H-H \rho)=\frac{1}{2}(L \rho+\rho L),
$$

we have

$$
\left\langle\psi_{m}|i \rho H-i H \rho| \psi_{n}\right\rangle=\frac{1}{2}\left\langle\psi_{m}|L \rho+\rho L| \psi_{n}\right\rangle .
$$

From this we have, for all $m$ and $n$,

$$
i\left(\lambda_{m}-\lambda_{n}\right)\left\langle\psi_{m}|H| \psi_{n}\right\rangle=\frac{\lambda_{m}+\lambda_{n}}{2}\left\langle\psi_{m}|L| \psi_{n}\right\rangle .
$$

Now,

$$
\begin{aligned}
I_{F}(\rho, H) & =\frac{1}{4} \operatorname{tr}\left(\rho L^{2}\right) \\
& =\frac{1}{4} \sum_{m}\left\langle\psi_{m}\left|\rho L^{2}\right| \psi_{m}\right\rangle \\
& =\frac{1}{4} \sum_{m, n} \lambda_{m}\left\langle\psi_{m}|L| \psi_{n}\right\rangle\left\langle\psi_{n}|L| \psi_{m}\right\rangle \\
& =\frac{1}{4} \sum_{m, n} \lambda_{m}\left|\left\langle\psi_{m}|L| \psi_{n}\right\rangle\right|^{2} .
\end{aligned}
$$

Symmetrically,

$$
I_{F}(\rho, H)=\frac{1}{4} \sum_{m, n} \lambda_{n}\left|\left\langle\psi_{m}|L| \psi_{n}\right\rangle\right|^{2} .
$$

Consequently,

$$
\begin{aligned}
I_{F}(\rho, H) & =\frac{1}{8} \sum_{m, n}\left(\lambda_{m}+\lambda_{n}\right)\left|\left\langle\psi_{m}|L| \psi_{n}\right\rangle\right|^{2} \\
& =\frac{4}{8} \sum_{m, n}\left(\lambda_{m}+\lambda_{n}\right) \frac{\left(\lambda_{m}-\lambda_{n}\right)^{2}}{\left(\lambda_{m}+\lambda_{n}\right)^{2}}\left|\left\langle\psi_{m}|H| \psi_{n}\right\rangle\right|^{2} \quad \text { (by Eq. } \\
& =\frac{1}{2} \sum_{m, n} \frac{1}{\lambda_{m}+\lambda_{n}}\left(\lambda_{m}-\lambda_{n}\right)^{2}\left|\left\langle\psi_{m}|H| \psi_{n}\right\rangle\right|^{2} \\
& =\frac{1}{2} \sum_{m, n}\left(1+\frac{2 \lambda_{m}^{1 / 2} \lambda_{n}^{1 / 2}}{\lambda_{m}+\lambda_{n}}\right)\left(\lambda_{m}^{1 / 2}-\lambda_{n}^{1 / 2}\right)^{2}\left|\left\langle\psi_{m}|H| \psi_{n}\right\rangle\right|^{2} .
\end{aligned}
$$

The conclusion of the theorem follows since

$$
0 \leq \frac{2 \lambda_{m}^{1 / 2} \lambda_{n}^{1 / 2}}{\lambda_{m}+\lambda_{n}} \leq 1, \quad \forall m, n .
$$

Remark. If $\rho$ commutes with $H$, then

$$
I_{W}(\rho, H)=I_{F}(\rho, H)=0 .
$$


Example. Let us consider a two-level quantum system. The quantum state Hilbert space is $\mathbb{C}^{2}$. A general density operator $\rho$ on $\mathbb{C}^{2}$ is of the form

$$
\rho=\frac{1}{2}\left(\begin{array}{cc}
1+r_{3} & r_{1}-i r_{2} \\
r_{1}+i r_{2} & 1-r_{3}
\end{array}\right)
$$

for some $\mathbf{r}=\left(r_{1}, r_{2}, r_{3}\right) \in \mathbb{R}^{3},|\mathbf{r}| \equiv \sqrt{r_{1}^{2}+r_{2}^{2}+r_{3}^{2}} \leq 1$. The eigenvalues of $\rho$ are $\lambda_{1}=\frac{1-|\mathbf{r}|}{2}$ and $\lambda_{2}=\frac{1+|\mathbf{r}|}{2}$. Let the corresponding eigenvectors be $\left|\psi_{1}\right\rangle$ and $\left|\psi_{2}\right\rangle$, then

$$
\rho=\lambda_{1}\left|\psi_{1}\right\rangle\left\langle\psi_{1}\left|+\lambda_{2}\right| \psi_{2}\right\rangle\left\langle\psi_{2}\right| .
$$

Consequently, by Eqs. (14) and (16),

$$
I_{W}(\rho, H)=\frac{1}{2}\left(1-\sqrt{1-|\mathbf{r}|^{2}}\right)\left|\left\langle\psi_{1}|H| \psi_{2}\right\rangle\right|^{2}, \quad I_{F}(\rho, H)=\frac{|\mathbf{r}|^{2}}{2}\left|\left\langle\psi_{1}|H| \psi_{2}\right\rangle\right|^{2}
$$

and

$$
I_{F}(\rho, H)=\left(1+\sqrt{1-|\mathbf{r}|^{2}}\right) I_{W}(\rho, H) .
$$

Thus $I_{F}(\rho, H)$ may vary continuously from $I_{W}(\rho, H)$ to $2 I_{W}(\rho, H)$. Moreover, in this case, if $\left\langle\psi_{1}|H| \psi_{2}\right\rangle \neq 0$ and $H$ does not commute with $\rho$, then $I_{W}(\rho, H)=$ $I_{F}(\rho, H)$ if and only if $|\mathbf{r}|=1$, that is, $\rho$ is a pure state.

\section{REFERENCES}

[1] S. I. Amari, Differential-Geometrical Methods in Statistics, Lecture Notes in Statistics, No. 28, Springer-Verlag, Berlin, 1985. MR 86m:62053

[2] N. N. Chentsov, Statistical Decision Rules and Optimal Inferences (in Russian), Nauka, Moscow, 1972. MR 49:8140

[3] A. Connes and E. Stormer, Homogeneity of the state space of factors of type $\mathrm{III}_{1}, J$. Funct. Anal. 28 (1978), 187-196. MR 57:10435

[4] A. Connes, Noncommutative Geometry, Academic Press, San Diego, CA, 1994. MR 95j: 46063

[5] H. Cramér, Mathematical Methods of Statistics, Princeton University Press, Princeton, New Jersey, 1946, thirteenth printing, pp. 477-481, 1974. MR 8:39f

[6] R. A. Fisher, Theory of statistical estimation, Proc. Cambridge Philos. Soc. 22 (1925), 700-725.

[7] B. R. Frieden, Physics from Fisher Information: A Unification, Cambridge University Press, 1998. MR 2000c: 81050

[8] C. W. Helstrom, Quantum detection and estimation theory, J. Statist. Phys. 1 (1969), 231252. MR 40:3855

[9] D. Petz, Monotone metrics on matrix spaces, Linear Algebra and its Applications, 244 (1996), 81-96. MR 97f: 15056

[10] E. P. Wigner and M. M. Yanase, Information contents of distributions, Proc. Nat. Acad. Sci. USA, 49 (1963), 910-918. MR 27:1113

[11] E. P. Wigner and M. M. Yanase, On the positive semidefinite nature of a certain matrix expression, Canadian J. Math. 16 (1964), 397-406. MR 29:114

Institute of Applied Mathematics, Academy of Mathematics and System Sciences, Chinese Academy of Sciences, Beijing, 100080 People's Republic of China

E-mail address: luosl@mail.amt.ac.cn 